\title{
The long-term efficacy of one-shot neoadjuvant intra-arterial chemotherapy combined with radical cystectomy versus radical cystectomy alone for bladder cancer: a propensity-score matching study
}

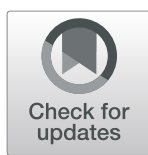

Wasilijiang Wahafu', Sai Liu', Wenbin Xu ${ }^{3}$, Mengtong Wang ${ }^{1}$, Qingbao $\mathrm{He}^{1}$, Liming Song ${ }^{1}$, Mingshuai Wang ${ }^{1}$, Feiya Yang ${ }^{1,2}$, Lin $\mathrm{Hua}^{3}$, Yinong $\mathrm{Niu}^{1 *}$ and Nianzeng Xing ${ }^{1,2^{*}}$

\begin{abstract}
Background: Bladder cancer is a complex disease associated with high morbidity and mortality. Management of bladder cancer before radical cystectomy continues to be controversial. We compared the long-term efficacy of one-shot neoadjuvant intra-arterial chemotherapy (IAC) versus no IAC (NIAC) before radical cystectomy (RC) for bladder cancer.

Methods: We performed a retrospective review of patients who underwent either one-shot IAC or NIAC before RC between October 2006 and November 2015. A propensity-score matching (1:3) was performed based on key characters. The Kaplan-Meier method was utilized to estimate survival probabilities, and the log-rank test was used to compare survival outcomes between different groups. A multivariable Cox proportional hazard model was used to estimate survival outcomes.
\end{abstract}

Results: Twenty-six patients were treated using IAC before RC, and 123 NIAC patients also underwent RC. After matching, there was no significant difference between groups in baseline characteristics, perioperative variables, complication outcomes or tumor characteristics. Compared with clinical tumor stages, pathological tumor stages demonstrated a significant decrease $(P=0.002)$ in the IAC group. There was no significant difference in overall survival (OS, $p=0.354$ ) or cancer-specific survival (CSS, $p=0.439)$ between the groups. Among all patients, BMl significantly affected OS $(p=0.004)$, and positive lymph nodes (PLN) significantly affected both OS $(p<0.001)$ and $\operatorname{CSS}(p=0.010)$.

Conclusions: One-shot neoadjuvant IAC before RC shows safety and tolerability and provides a significant advantage in pathological downstaging but not in OS or CSS. Further study of neoadjuvant combination therapeutic strategies with $\mathrm{RC}$ is needed.

Keywords: Bladder cancer, Neoadjuvant chemotherapy, Intra-arterial infusion, Cystectomy, Treatment outcome

\footnotetext{
*Correspondence: 18601020160@163.com; nianzengxing@yeah.net

'Institute of Urology, Capital Medical University, Department of Urology,

Capital Medical University Beijing Chao-Yang Hospital, Beijing 100020, China

Full list of author information is available at the end of the article
}

(c) The Author(s). 2019 Open Access This article is distributed under the terms of the Creative Commons Attribution 4.0 International License (http://creativecommons.org/licenses/by/4.0/), which permits unrestricted use, distribution, and reproduction in any medium, provided you give appropriate credit to the original author(s) and the source, provide a link to the Creative Commons license, and indicate if changes were made. The Creative Commons Public Domain Dedication waiver (http://creativecommons.org/publicdomain/zero/1.0/) applies to the data made available in this article, unless otherwise stated. 


\section{Background}

Bladder cancer is a complex disease associated with high morbidity and mortality rates. Approximately $75 \%$ of newly diagnosed patients present with non-muscleinvasive bladder cancer (MIBC), which is characterized by a high recurrence rate and 5-yr survival of 90\% [1]. Once the disease becomes MIBC, the 5-year overall survival is a dismal outcome at $47 \%$ compared with the $81 \%$ survival rate of patients with non-muscle-invasive disease [2]. Approximately 50\% of MIBC patients will develop metastasis and have a 5 -yr survival of only $~ 5 \%$ $[3,4]$. Despite radical cystectomy (RC) with bilateral pelvic lymph node dissection (PLND) as the gold standard treatment, $\mathrm{RC}$ only permits a 5 -yr survival in approximately $50 \%$ of patients $[3,5-8]$. In fact, there was no significant improvement in bladder cancer outcomes over the last three decades.

Although several high-quality clinical trials have demonstrated improved survival and pathologic downstaging with the use of chemotherapy prior to $\mathrm{RC}$, adoption of neoadjuvant chemotherapy for MIBC has been slow. Several hypotheses, such as the significant toxicities and delayed surgery, especially the inability to identify which patients could derive the most benefit from neoadjuvant chemotherapy, were slow during the adoption of neoadjuvant treatment. Additionally, 25 to $33 \%$ of patients are unable to receive adjuvant chemotherapy after $\mathrm{RC}$ due to postoperative problems, such as perioperation complications or deterioration of renal function $[9,10]$. Therefore, we hypothesized that one-shot neoadjuvant intraarterial chemotherapy (IAC) would have less toxicity and better disease control than RC alone. Moreover, this strategy would allow patients to complete therapy quickly and move on to the next form of therapy.

Therefore, we compared the long-term efficacy of oneshot neoadjuvant IAC versus no IAC (NIAC) before RC for bladder cancer in this study.

\section{Methods}

To evaluate the long efficacy of one-shot neoadjuvant IAC versus NIAC before RC for bladder cancer, we retrospectively reviewed all patients treated with $\mathrm{RC} /$ PLND between October 2006 and November 2015 for urothelial carcinoma of the bladder without distant metastasis in the Department of Urology, Beijing ChaoYang Hospital. This study was approved by the Institutional Review Board of Beijing Chao-Yang Hospital. To prevent selection bias of the learning curve, we chosen patients who operations were performed by the same laparoscopic surgeon (Xing).

\section{Patient eligibility and selection}

The diagnosis of bladder cancer was made using imaging findings (ultrasonography, computed tomography, magnetic resonance imaging), chest radiography with or without cystoscopic biopsy, and routine laboratory analysis. The TNM classification was staged according to the American Joint Committee on Cancer staging system (7th 2010). Clinical staging was based on the physical examination, imaging findings, and biopsies of bladder tumors before the start of therapy. All patients had pathologic documentation of urothelial carcinoma, which was defined as local disease (pT2-4 N0/ $+\mathrm{M} 0$ ) or non-muscle-invasive bladder cancer (NMIBC), but the patients were at high risk for tumors [T1G3 with concurrent carcinoma in situ (CIS) at diagnosis, multiple and/or large T1G3, recurrent T1G3]. The pathological results were reviewed by the two genitourinary pathologists after matching the two groups. Patient with pelvic lymph node metastasis diagnosed by imaging studies were eligible. Patients who underwent neoadjuvant intravesicle chemotherapy but not adjuvant chemotherapy were ineligible. Patients who had nonurothelial carcinoma $(n=11)$, preoperative pelvic irradiation $(n=5)$, missing clinical information $(n=11)$ or who were lost during follow-up $(n=17)$ were excluded, leaving 149 patients available for analysis.

\section{IAC treatment protocol}

Gemcitabine $\left(700-1000 \mathrm{mg} / \mathrm{m}^{2}\right)$ and cisplatin $(35-70$ $\mathrm{mg} / \mathrm{m}^{2}$ ) were infused into the femoral artery to the internal iliac artery using the Seldinger technique. The approach of 15 patients was from the bilateral internal iliac artery, while the unilateral internal iliac artery was used in 11 patients, and the approach was based on tumor location as determined by imaging tests, cystoscopy and digital subtraction angiography. Complete blood counts and biochemical studies were performed every 2 weeks. Patients were evaluated for treatment responses using imaging tests and were assigned to receive $\mathrm{RC} / \mathrm{PLND} 4$ weeks after IAC to allow adequate recovery.

\section{Statistical analysis \\ Baseline comparison between the intra-arterial and no intra-arterial groups}

Key baseline characteristics [gender, age, Body mass index (BMI), hypertension, diabetes, age-adjusted Charlson comorbidity index (CCI), American Society of Anesthesiologists (ASA) score, Eastern Cooperative Oncology Group performance status (ECOG PS), smoking history, time between tumor confirmation and $\mathrm{RC}$, preoperative irradiations, and follow-up duration)]were compared between the IAC and NIAC groups.

Continuous characters were compared by independent sample t-tests when the data were normally distributed and by Wilcoxon rank sum test when the data were nonnormally distributed. The Pearson chi-square test or Fisher's exact test was performed to calculate $p$ values for categorical factors. The Wilcoxon rank sum test was performed to compare ordinal values. 


\section{Propensity-score matching}

We performed matched group analysis to control for differences between groups due to selection bias and confounding factors. Propensity-score matching was performed based on key characters, including gender, age, BMI, hypertension, diabetes, age-adjusted CCI, ASA score, ECOG PS, smoking history, time between tumor confirmation and RC, preoperative irradiations and follow-up duration. Propensity scores were estimated using a logistic regression model. A 1:3 matching with no replacement was applied using the nonrandom package in R (http://www.r-project.org). A t-test or Wilcoxon rank sum test, or Pearson's chi-square test or Fisher's exact test, was applied to compare differences in covariates after matching to demonstrate that matching enhanced the balance between groups.

\section{Oncological outcomes in the matched group}

We compared oncological outcomes in a matched cohort using a t-test, a Wilcoxon rank sum test, Pearson's chi-square tests and Fisher's exact test. The KaplanMeier method was utilized to estimate survival probabilities, and the log-rank test was used to compare survival outcomes between different groups. A multivariable Cox proportional hazard model was used to estimate survival outcomes.

All statistical analyses, except for propensity-score matching, were performed with IBM SPSS version 19.0 (IBM corp., Armonk, NY). Statistical significance was considered at two-sided $p<0.05$. All statistical plots were drawn in GraphPad prism version 6.0 (GraphPad Software Inc., La Jolla, CA 92037 USA).

\section{Results}

A total of 26 patients underwent one-shot neoadjuvant IAC, and 123 patients were treated using RC/PLND alone. The baseline characteristics of patients enrolled are listed in Table 1. All key variables except follow-up duration ( 88 mo vs 26 mo, $p=0.002$ ) were not different at baseline between the two groups. To reduce the differences between groups due to selection bias, we performed a matched analysis based on follow-up duration Additional file 2 Figure S1.

The matching algorithm was 1:3, which was the optimal weight for each key variable. The patients were followed up for a median period of 88 months in the IAC group and for 56 months in the NIAC group ( $p=$ 0.161 ). There were no significant differences between the groups in patient demographics and clinical characteristics. Table 1 lists the baseline characteristics for the matched cohorts.

There was no significant difference in perioperative variables between the IAC and NIAC groups (Table 2). In the type of urinary diversion, more than $50 \%$ of patients received orthotopic neobaldders in both groups. IAC treatment did not affect renal function in terms of serum creatinine $(P=0.702)$ or blood urea nitrogen $(P=$ $0.119)$ levels. The proportion of those who remained in

Table 1 Baseline characteristics of the patients in the IAC and NIAC before and after matched groups (1:3)

\begin{tabular}{|c|c|c|c|c|c|}
\hline & \multirow[t]{2}{*}{ Intra-arterial } & \multirow{2}{*}{$\begin{array}{l}\text { Before matched groups } \\
\text { No intra-arterial }\end{array}$} & \multicolumn{3}{|c|}{ After matched groups $(1: 3)$} \\
\hline & & & $p$ value & No intra-arterial & $\mathrm{p}$ value \\
\hline Patients (n) & 26 & 123 & & 78 & \\
\hline Gender & & & 1.000 & & 1.000 \\
\hline Female, n (\%) & $4(15.4 \%)$ & 19(15.4\%) & & $10(12.8 \%)$ & \\
\hline Male, n (\%) & $22(84.6 \%)$ & 104(84.6\%) & & $68(87.2 \%)$ & \\
\hline Age, yr, median (IQR) & $60.0(55.0-71.0)$ & $63.0(56.0-72.0)$ & 0.328 & $62.5(56.0-69.3)$ & 0.799 \\
\hline Body mass index (kg/m2) & $25.2 \pm 3.12$ & $24.1 \pm 3.8$ & 0.184 & $24.3 \pm 3.1$ & 0.202 \\
\hline Hypertension, n (\%) & $12(46.2 \%)$ & $38(30.9 \%)$ & 0.134 & $27(34.6 \%)$ & 0.293 \\
\hline Diabetes, n (\%) & $4(15.4 \%)$ & $16(13.0 \%)$ & 0.995 & $8(10.3 \%)$ & 0.723 \\
\hline Age-adjusted CCl & $4.0(3.0-7.0)$ & $4.0(3.0-5.0)$ & 0.625 & $4.0(3.0-6.0)$ & 0.909 \\
\hline ASA score & $2.0(1.8-2.0)$ & $2.0(2.0-2.0)$ & 0.221 & $2.0(2.0-2.0)$ & 0.188 \\
\hline ECOG PS & $1.0(0.0-1.0)$ & $1.0(0.0-1.0)$ & 0.490 & $1.0(1.0-1.0)$ & 0.394 \\
\hline Smoking history, n (\%) & $15(57.7 \%)$ & $58(47.2 \%)$ & 0.329 & $40(51.3 \%)$ & 0.571 \\
\hline Time between confirmed tumor and RC, mo, median (IQR) & $3.0(1.0-6.8)$ & $5.0(1.0-18.0)$ & 0.133 & $5.0(1.0-18.0)$ & 0.173 \\
\hline TURBT before RC & $7(25.9 \%)$ & $57(46.3 \%)$ & 0.048 & $32(41.0 \%)$ & 0.100 \\
\hline Preoperative irradiation, $\mathrm{n}(\%)$ & $0(0.0 \%)$ & $5(4.1 \%)$ & 0.587 & $3(3.8 \%)$ & 0.571 \\
\hline Follow-up length, mo, median (IQR) & $88.0(37.0-109.0)$ & $26.0(14.0-65.0)$ & 0.002 & $56.0(30.8-91.3)$ & 0.161 \\
\hline
\end{tabular}

IAC, intra-arterial chemotherapy; NIAC, no-intra-arterial chemotherapy; IQR = interquartile range; $\mathrm{RC}=$ radical cystectomy; $\mathrm{ASA}=\mathrm{American}$ Society of Anesthesiologists; $\mathrm{CCl}=$ Charlson comorbidity index; ECOG PS = Eastern Cooperative Oncology Group performance status 
Table 2 Perioperative variables of the matched groups

\begin{tabular}{|c|c|c|c|}
\hline & Intra-arterial & No Intra-arterial & $\mathrm{p}$ value \\
\hline Patients (n) & 26 & 78 & \\
\hline Type of urinary diversion, $\mathrm{n}(\%)$ & & & 0.840 \\
\hline Cutaneous ureterostomy & $2(7.7 \%)$ & $5(6.4 \%)$ & \\
\hline lleal conduit & $9(34.6 \%)$ & $32(41.0 \%)$ & \\
\hline Orthotopic neobladder & $15(57.7 \%)$ & $41(52.6 \%)$ & \\
\hline Operating time, min, mean (IQR) & $369.0(300.0-420.0)$ & $382.9(306.0-420.0)$ & 0.574 \\
\hline Estimated blood loss, ml, mean (IQR) & $411.5(187.5-525.0)$ & $348.1(200.0-400.0)$ & 0.456 \\
\hline Removed Jackson-Pratt drain, day, mean (IQR) & 12.6(9.0-14.3) & 14.7(8.0-19.0) & 0.591 \\
\hline Passing flatus, day, mean (IQR) & $4.9(3.0-6.0)$ & $4.0(3.0-5.0)$ & 0.189 \\
\hline Adjuvant chemotherapy, n (\%) & $4(15.4 \%)$ & $12(15.4 \%)$ & 1.000 \\
\hline \multicolumn{4}{|l|}{ Pre-op laboratory studies } \\
\hline HGB (g/L), median (IQR) & 134.0(122.3-142.3) & 132.5(119.8-146.3) & 0.943 \\
\hline HCT (\%), median (IQR) & $38.6(36.9-41.5)$ & $39.8(36.1-42.4)$ & 0.615 \\
\hline WBC, median (IQR) & $6.4(5.0-7.6)$ & $6.5(5.3-7.8)$ & 0.286 \\
\hline Platelets, median (IQR) & 218.5(193.0-262.5) & 216.5(187.3-258.5) & 0.768 \\
\hline BUN(mmol/L), median (IQR) & $5.7(4.7-7.0)$ & $6.1(4.6-8.0)$ & 0.119 \\
\hline Creatinine $(\mu \mathrm{mol} / \mathrm{L})$, median (IQR) & $84.2(70.3-113.5)$ & 82.2(70.1-99.9) & 0.702 \\
\hline Albumin (g/L), median (IQR) & $35.1(32.8-39.0)$ & $36.0(33.0-39.9)$ & 0.931 \\
\hline Overall complications, n (\%), Clavien grade & $24(92.3 \%)$ & 75(96.2\%) & 0.791 \\
\hline Perioperative complications (< $30 \mathrm{~d}), \mathrm{n}(\%)$, & & & 0.930 \\
\hline 0 & $2(7.7 \%)$ & $3(3.8 \%)$ & \\
\hline 1 & $0(0.0 \%)$ & $2(2.6 \%)$ & \\
\hline 2 & $21(80.8 \%)$ & $66(84.6 \%)$ & \\
\hline 3 & $3(11.5 \%)$ & $5(6.4 \%)$ & \\
\hline 4 & $0(0.0 \%)$ & $0(0.0 \%)$ & \\
\hline Short-term complications (< 90 d), n (\%) & & & 0.516 \\
\hline 0 & $24(92.3 \%)$ & $68(87.2 \%)$ & \\
\hline 1 & $0(0.0 \%)$ & $4(5.1 \%)$ & \\
\hline 2 & $1(3.8 \%)$ & $3(3.8 \%)$ & \\
\hline 3 & $1(3.8 \%)$ & $3(3.8 \%)$ & \\
\hline 4 & $0(0.0 \%)$ & $0(0.0 \%)$ & \\
\hline Long-term complications (>90 d), n (\%) & & & 0.616 \\
\hline 0 & $24(92.3 \%)$ & $74(94.9 \%)$ & \\
\hline 1 & $0(0.0 \%)$ & $0(0.0 \%)$ & \\
\hline 2 & $0(0.0 \%)$ & $0(0.0 \%)$ & \\
\hline 3 & $1(3.8 \%)$ & $3(3.8 \%)$ & \\
\hline 4 & $1(3.8 \%)$ & $1(1.3 \%)$ & \\
\hline Surgery intensive care unit stay, n (\%) & $0(0.0 \%)$ & $8(10.3 \%)$ & 0.196 \\
\hline
\end{tabular}

$\mathrm{IQR}=$ interquartile range; $\mathrm{HGB}=$ hemoglobin; $\mathrm{HCT}=$ hematocrit; $\mathrm{WBC}=$ white blood cell; $\mathrm{BUN}=$ blood urea nitrogen

the intensive care unit after surgery was lower in the IA group than in the NIAC group (0\% vs $10.3 \% ; p=0.196)$. The total complication rate was not significantly different between the two groups (92.3\% vs $96.2 \% ; p=0.791)$. However, Clavien grade 2 complications $(>80 \%)$ were more common in the perioperative period $(<30 \mathrm{~d})$.
Tumor characteristics are listed in Table 3. The pathology results of all patients showed urothelial cell carcinoma of the urinary bladder. Positive surgical margins were reported in the NIAC group (3.8\%). Compared with clinical TNM stages, pathological TNM staging demonstrated similar in the NIAC group after matching 
Table 3 Tumor characteristics of the matched groups

\begin{tabular}{|c|c|c|c|}
\hline & Intra-arterial & No intra-arterial & $\mathrm{p}$ value \\
\hline Patients (n) & 26 & 78 & \\
\hline Pathologic stage outcome, n (\%) & & & 0.414 \\
\hline pT1 & $9(34.6 \%)$ & $26(33.3 \%)$ & \\
\hline pT2a & $6(23.1 \%)$ & 11(14.1\%) & \\
\hline pT2b & $1(3.8 \%)$ & $14(17.9 \%)$ & \\
\hline pT3a & $6(23.1 \%)$ & $11(14.1 \%)$ & \\
\hline pT3b & $3(11.5 \%)$ & $3(3.8 \%)$ & \\
\hline pT4a & $1(3.8 \%)$ & $13(16.7 \%)$ & \\
\hline Histology grade, n (\%) & & & 0.566 \\
\hline Low grade & $6(23.1 \%)$ & $14(17.9 \%)$ & \\
\hline High grade & $20(76.9 \%)$ & $64(82.1 \%)$ & \\
\hline Pathology, n (\%) & & & 0.399 \\
\hline Urothelial cancer & $21(80.8 \%)$ & $71(91.0 \%)$ & \\
\hline Urothelial cancer with squamous differentiation & $3(11.5 \%)$ & $4(5.1 \%)$ & \\
\hline Urothelial cancer with glandular differentiation & $2(7.7 \%)$ & $3(3.8 \%)$ & \\
\hline Nodes removed, median (IQR) & $17.0(11.8-21.3)$ & $14.0(8.0-19.0)$ & 0.304 \\
\hline PLN, median (range) & $0.0(6.0)$ & $0.0(27.0)$ & 0.904 \\
\hline Lymph-node-positive patients, n (\%) & $7(26.9 \%)$ & $18(23.1 \%)$ & 0.691 \\
\hline Positive surgical margins, n (\%) & $0(0.0 \%)$ & $3(3.8 \%)$ & 0.571 \\
\hline Associated CIS, no. (\%) & $4(15.4 \%)$ & $12(15.4 \%)$ & 1.000 \\
\hline
\end{tabular}

$\mathrm{QQR}=$ interquartile range; $\mathrm{CIS}=$ carcinoma in situ; $\mathrm{PLN}=$ positive lymph nodes

$(P=0.519$, Additional file 1 Table S1 and Additional file 2 Figure S2); however, a significant decrease showed in the IAC group $(P=0.002): 7(26.9 \%)$ patients had no stage change, 17 (65.4\%) patients exhibited a stage decrease, and 2 (7.7\%) patients exhibited a stage increase (Additional file 1 Table S2 and Additional file 2 Figure S3). There was one patient with severe gross hematuria that was diagnosed as NMIBC by CT. Conservative measures and attempts to achieve hemostasis by cystoscopy were unsuccessful at controlling bleeding. The patient therefore underwent endovascular treatment with intra-arterial chemotherapy and superselective embolization of the vesical arteries 2 weeks before RC/PLND.

Of the 26 patients in the IAC group, two (7.7\%) died because of cancer, and one $(3.8 \%)$ died due to another reason. Among the 78 patients in the NIAC group, eleven (14.1\%) suffered cancer-specific mortality, and five (6.4\%) died due to another reason. There was no significant difference in the rates along the curve for overall mortality $(p=0.354)$ or cancerspecific mortality $(p=0.439)$ between the IAC and NIAC groups (Fig. 1).
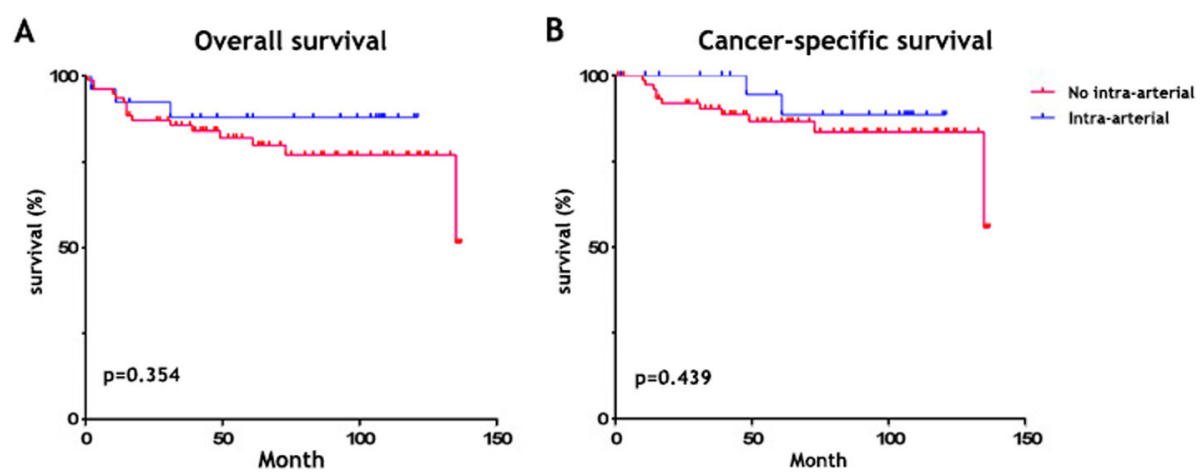

Fig. 1 Overall survival and cancer-specific survival. a Three (11.5\%) and sixteen (20.4\%) patients died in the IAC and NIAC groups, respectively $(p=0.354)$. b Two $(7.7 \%)$ and eleven (14.1\%) patients suffered cancer-specific mortality in the IAC and NIAC groups, respectively $(p=0.439)$ 
Multivariable Cox proportional hazards regression analysis (Table 4) showed that several variables have an impact on overall survival. In all samples, BMI $(p=$ $0.005)$, diabetes $(p=0.002)$, ASA score $(\mathrm{p}=0.005)$, PLN $(\mathrm{p}<0.001)$ and perioperative complications $(p=0.020)$ were influencing factors.

When these potential factors were used to calculate the Kaplan-Meier survival curve, some were associated with OS and CSS (Fig. 2). BMI less than $25 \mathrm{~kg} / \mathrm{m}^{2}$ was associated with OS $(p=0.004)$ but not CSS $(p=0.050)$, and PLN was associated with OS $(\mathrm{p}<0.001)$ and CSS $(p=$ $0.010)$. The survival time and cumulative survival rate (1-, 5- and 10-year rates) are depicted in Table 5.

\section{Discussion}

Our present results show that there was a downstaging advantage with one-shot neoadjuvant IAC before $\mathrm{RC}$ for MIBC ( $\mathrm{p}=0.002)$, but it did not significantly improve OS $(p=0.354)$ or CSS $(p=0.439)$ compared to those treated without IAC. We performed Cox regression to assess risk factors association with survival in all samples and found that BMI (less than a $25 \mathrm{~kg} / \mathrm{m}^{2}$ ) significantly affected OS $(\mathrm{p}=0.004)$, and PLN significantly affected both OS $(\mathrm{p}<0.001=$ and CSS $(\mathrm{p}=0.010)$. Besides, we are curious about the potential risk factors affecting survival outcomes in the IAC and NIAC group and their difference in the two groups. So, despite the small sample size of IAC and NIAC group, we used Cox regression to explore the risks in the both groups exploratorily. The exploratory analysis found that diabetes $(P=0.029, \mathrm{RR}=$ 14.649) was an influencing factor in IAC group, whereas BMI $(P=0.015, \mathrm{RR}=0.802), \mathrm{PLN}(P<0.001, \mathrm{RR}=7.474)$ and smoking history $(P=0.043, \mathrm{RR}=3.388)$ were influencing factors in NIAC group (Additional file 1 Table S3). Furthermore, when these potential factors were used to calculate the Kaplan-Meier survival curve, some were associated with OS and CSS in IAC groups and NIAC groups (Additional file 1 Table S4-S7 and Additional file 2 Figure S4). In brief, one-shot neoadjuvant resulted in significant downstaging; for RC, only BMI and PLN correlate with survival in our long-term data.

RC usually occurs 4 to 6 weeks after MIBC diagnosis in our center, and this time offers an opportunity to

Table 4 Multivariable Cox proportional hazards model to estimate survival outcomes

\begin{tabular}{lll}
\hline Variables & \multicolumn{2}{l}{ Total } \\
\cline { 2 - 3 } & p value & RR $(95 \% \mathrm{Cl})$ \\
\hline BMI & 0.005 & $0.767(0.638-0.922)$ \\
Diabetes & 0.002 & $8.716(2.263-33.563)$ \\
ASA score & 0.005 & $4.846(1.600-14.682)$ \\
Positive lymph nodes & $<0.001$ & $11.886(3.912-36.119)$ \\
Perioperative complication & 0.020 & $4.416(1.259-15.488)$ \\
\hline
\end{tabular}

preoperatively perform neoadjuvant therapy. Although standard neoadjuvant cisplatin-based combination chemotherapy followed by $\mathrm{RC}$ is supported by level 1 evidence for resectable (cT2-T4aNOM0) MIBC, the inability to identify which patients may derive most benefit from neoadjuvant chemotherapy was slow during the adoption of neoadjuvant treatment. Nevertheless, approximately $50 \%$ of patients with urothelial carcinoma are considered ineligible to receive cisplatin based on renal dysfunction and impaired performance status, and a subset of patients also refuse to receive neoadjuvant chemotherapy [11]. Notably, adherence to adjuvant and neoadjuvant chemotherapy regimens was observed in a similarly low proportion of patients (approximately 21\% each) in the USA, and the majority of patients with resectable bladder cancer received no chemotherapy at all [12]. Therefore, the treatment algorithm for MIBC tumors in a short window before RC is still evolving.

Neoadjuvant IAC is not a new concept. In the 1980s1990s, multiple efforts were made to improve oncological outcomes by adding various IAC treatment modalities plus $\mathrm{RC}$ to treatment regimens for MIBC. A summary of the published neoadjuvant IAC papers, including key information on chemotherapy regimens, is provided in Table 6 (Additional file 2 Figure S5) [13-21]. Although most of the literature is early in its use, the drugs also have differences, but all show varying degrees of pathological downstaging or even complete response (CR; pT0). Pathological downstaging or pathological $\mathrm{CR}$ to neoadjuvant chemotherapy is a well-recognized biomarker of improved OS [22]. Because it was such a short period of therapy, we felt that achieving pathological CR would be quite challenging in our study. Although OS and CSS for the study cohort remained disappointing, one-shot neoadjuvant IAC showed an encouraging pathological downstaging rate of greater than $60 \%(P=0.002)$. Meanwhile, the safety and tolerability profile for IAC was quite favorable. In particular, no chemotherapy-related adverse events have been reported in the IAC group, which did not delay planned surgery. Moreover, no differences in perioperative, shortterm or long-term complications were recorded compared with patients undergoing RC only. Similarly, intraoperative performance (operating time, estimated blood loss, blood transfusion, number of nodes removed and surgical margins) was not compromised by neoadjuvant IAC. Therefore, our treatment produced major pathologic responses, indicating that the side effects of chemotherapy can be reliably avoided when using one-shot IAC.

Bladder cancer is a heterogeneous disease, which means that only single treatment is not enough. Current research is actively exploring novel combinations and ideal sequencing with various treatment modalities, especially immunotherapy combined with chemotherapy, radiotherapy or targeted therapies. Although bladder 


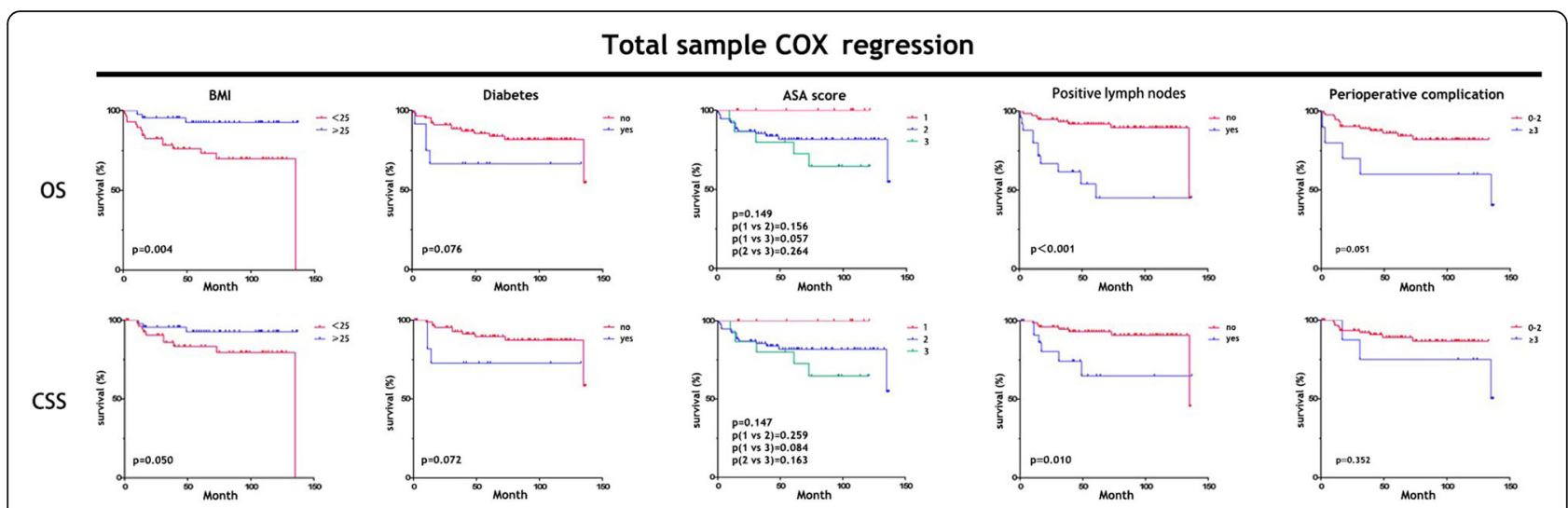

Fig. 2 Overall survival and cancer-specific survival from Cox proportional hazards regression analysis (see Table 5). BMl less than $25 \mathrm{~kg} / \mathrm{m}^{2}$ was associated with OS $(p=0.004)$ but not CSS $(p=0.050)$, and PLN was associated with OS $(p<0.001)$ and CSS $(p=0.010)$ in all sample groups

cancer carries the third highest mutation rate of all studied cancers, suggesting the possibility of increased immunogenicity via the development of neoantigens, it is clear from existing data that the majority of patients will not respond to monotherapy [23-25]. Interestingly, chemotherapeutic agents have direct cytotoxic effects on tumor cells that release tumor antigens but also have positive effects on immune effector $\mathrm{T}$ cells [26]. Therefore, in theory, one-shot IAC can play a synergistic role as a single immunotherapy. Moreover, chemotherapy may substantially prolong the total duration of neoadjuvant immunotherapy [27]. However, patient selection must be optimized. In addition to having good renal function, it is also necessary to pay attention to the patient's nutritional status and immune system, which may be hampered by an aged-related reduction in functional decline. With an average age of 73 years at diagnosis, perioperative immunonutrition has a significant impact on surgery and the efficacy of immunotherapy [28]. According to our findings, BMI, diabetes and ASA score were associated with survival and may be the modifiable predictors in older and sicker patient populations.
Additionally, the optimization of toxicity and tolerability of combination therapies through appropriate dosing and sequencing should be determined using welldesigned clinical trials.

The strengths of our study are the selection of only one surgeon's cases for minimizing the influence of different levels of maturity and the use of propensity-score matching to reduce the inherent biases. As a result, patients who were matched only on the basis of key variables were selected. However, an important limitation is our drawing conclusions from small sample and highly selected patients with retrospective, nonrandomized data, which might introduce possible selection biases that we did not control for. Another limitation of the present study was that there was no consistent record of recurrence-free survival (RFS) in the long-term followup period. Although the final pathology showed no difference between the cohorts, the proportion of positive surgical margins was higher in NIAC cohort. It is possible that NIAC cohort had lower stage disease to begin with which would affect the RFS of patients. However, it should be noted that OS is the gold standard and the

Table 5 Description of survival of groupings in the entire set of patients (see Fig. 2)

\begin{tabular}{|c|c|c|c|c|c|}
\hline & Mean ST (mo) & Medium ST (mo) & 1-year CSR (95\%CI) & 5-year CSR (95\%Cl) & 0-year CSR (95\%Cl) \\
\hline \multicolumn{6}{|c|}{ OS of BMI grouping } \\
\hline$<25$ & 102.35 & 135.00 & $0.897(0.784-0.952)$ & $0.733(0.587-0.835)$ & $0.699(0.541-0.811)$ \\
\hline$\geq 25$ & 129.00 & - & $0.956(0.0-1.0)$ & $0.927(0.0-1.0)$ & $0.927(0.0-1.0)$ \\
\hline \multicolumn{6}{|c|}{ OS of PLN grouping } \\
\hline No & 124.81 & 135.00 & $0.975(0.903-0.994)$ & $0.920(0.830-0.963)$ & $0.897(0.793-0.951)$ \\
\hline Yes & 75.65 & 61.00 & $0.800(0.000-1.000)$ & $0.540(0.002-0.943)$ & $0.450(0.006-0.884)$ \\
\hline \multicolumn{6}{|c|}{ CSS of PLN grouping } \\
\hline No & 126.37 & 135.00 & $0.987(0.913-0.998)$ & $0.932(0.843-0.971)$ & $0.909(0.804-0.959)$ \\
\hline Yes & 98.10 & - & $0.861(0.000-1.000)$ & $0.649(0.000-0.985)$ & $0.649(0.000-0.985)$ \\
\hline
\end{tabular}

ST: survival time; CSR: cumulative survival rate; OS: overall survival; CSS: cancer-specific surival; BMI: body mass index; PLN: positive lymph nodes 
Table 6 Summary of the published papers on neoadjuvant intra-arterial chemotherapy followed by radical cystectomy

\begin{tabular}{|c|c|c|c|c|c|c|c|c|c|}
\hline Study & Year & Country & $\begin{array}{l}\text { Type of } \\
\text { study }\end{array}$ & $\begin{array}{l}\text { Sample size } \\
\text { (RC/total) }\end{array}$ & $\begin{array}{l}\text { Chemotherapy } \\
\text { regimen }\end{array}$ & $\begin{array}{l}\text { No. of } \\
\text { cycles }\end{array}$ & $\begin{array}{l}\text { Interval to } \\
\text { RC, (wks.) }\end{array}$ & $\begin{array}{l}\text { Downstaging, } \\
(\%) \text {, only RC }\end{array}$ & OS (only RC) \\
\hline Kanoh et al. [13] & 1983 & Japan & Retrospective & $7 / 13$ & ADM & $\begin{array}{l}\text { 2/wk. } \\
(\geq 3 \text { wks) }\end{array}$ & 6.7 & $5(71.4)$ & 2 died (14.6) \\
\hline Kamidono et al. [14] & 1984 & Japan & Retrospective & $11 / 11$ & ADM, MMC & 1 & 4.2 & $7(63.6)$ & 3 died (17.5) \\
\hline Maatman et al. [15] & 1986 & Italia & Prospective & $16 / 25$ & CDDP, ADM & $1-4$ & 4 & $4(25)$ & 1 died (15.7) \\
\hline Kanoh et al. [16] & 1987 & Japan & Retrospective & $15 / 32$ & $\mathrm{ADM} \pm \mathrm{CDDP}$ & $\begin{array}{l}10-23 \\
(17)\end{array}$ & - & - & 1 died, 5-year OS 87.5\% \\
\hline Kakizaki et al. [17] & 1987 & Japan & Retrospective & $29 / 29$ & $\begin{array}{l}\text { MMC, CPM, } \\
\text { thio-TEPA, 5-FU, } \\
\text { ADM, CDDP }\end{array}$ & 1 & 2 & - & - \\
\hline Jacobs et al. [18] & 1989 & USA & Retrospective & $16 / 30$ & CDDP & 1 & 4 & $15(93.8)$ & $\begin{array}{l}3 \mathrm{~N}+\text { average } 13 \mathrm{mo} 8 \\
\mathrm{NO} \text { average } 28 \pm 8 \mathrm{mo}\end{array}$ \\
\hline Galetti et al. [19] & 1989 & USA & Phase II & 4/8(only IA) & CDDP & 1 & - & $3(75)$ & $37 \mathrm{mo}(6-56)$ \\
\hline Arima et al. [20] & 1997 & Japan & Retrospective & $80 / 120$ & $\mathrm{ADM}+\mathrm{CDDP}$ & $1-4$ & - & $75(62.5)$ & - \\
\hline Miyata et al. [21] & 2015 & Japan & Retrospective & $17 / 50$ & $\begin{array}{l}\text { CDDP, ADM, } \\
\text { EPI }\end{array}$ & $2 \pm 0.2$ & $4-8$ & - & - \\
\hline Recent study & 2019 & China & Retrospective & $26 / 26$ & GC & 1 & 4 & $17(65.4)$ & $\begin{array}{l}3 \text { died (2 from cancer: } \\
11 \text { and } 31 \mathrm{mo})\end{array}$ \\
\hline
\end{tabular}

$\mathrm{RC}$, radical cystectomy; OS, overall survival; ADM, adriamycin or doxorubicin; MMC, mitomycin C; CDDP, cisplatin; EPI, epirubicin; GC, gemcitabine + cisplatin; - , not available

most dependable end point in clinical cancer research to support treatment algorithms. Furthermore, CSS may be a surrogate endpoint for RFS. Nevertheless, we were not able to detect statistically significant differences between the groups in OS or CSS. At the same time, more than half of our patients were from all over the country, and some proportion of patients did not have clear data on disease recurrence. Therefore, RFS is not as important. Finally, we should know that there was not a specific marker to judge the safety, tolerability, or clinical benefit of the treatments in the subgroups of patients. Answers to some of these questions will become clearer as these studies begin to mature with clinical readouts.

\section{Conclusions}

This long-term follow-up, retrospective study of one-shot neoadjuvant IAC in patients who underwent RC from 2006 to 2015 shows significant advantages in pathological downstaging but not in OS or CSS. Moreover, this study demonstrates the safety and tolerability of this treatment and provides a basis for combination therapy. Future efforts to improve survival in patients with bladder cancer is warranted and further study of the ideal neoadjuvant therapeutic strategies followed by RC is needed.

\section{Supplementary information}

Supplementary information accompanies this paper at https://doi.org/10. 1186/s12894-019-0552-7.

Additional file 1: Table S1. Pathological staging before and after surgery in the NIAC group after matching (see Fig. S2), Table S2.
Pathological staging before and after surgery in the IAC group (see Fig. S3), Table S3. Multivariable Cox proportional hazard model to estimate survival outcomes in IAC and NIAC groups, Table S4. Description of OS of diabetes groupings in the IAC group (see Fig. S4A), Table S5.

Description of OS of BMI groupings in the NIAC group (see Fig. S4B),

Table S6. Description of OS of PLN groupings in the NIAC group (see Fig. S4B), Table S7. Description of CSS of PLN groupings in the NIAC group (see Fig. S4B)

Additional file 2: Figure S1. Propensity-score matching analysis based on follow-up duration (Box plot), (A), Distribution of different groups of patients by follow-up time before the match (B), Distribution of different groups of patients by follow-up time after 1:3 matching, Figure S2. Tumor staging changes in the NIAC group after matching (see Table S1), Figure S3. Tumor staging changes in the IAC group (see Table S2), Figure S4. Overall survival and cancer-specific survival from Cox proportional hazards regression analysis (see Table S3-S6), (A), Diabetes was associated with only OS $(p=0.004)$ in the IAC group. (B). BMI was only associated with OS $(p=0.014)$, and PLN was associated with both OS ( $p<$ $0.001=$ and CSS $(p=0.017)$ in the NIAC group, Figure S5. Flow diagram of the article selection process

\section{Abbreviations}

ADM: adriamycin or doxorubicin; ASA: American Society of Anesthesiologists; BMI: body mass index; BUN: blood urea nitrogen; CCl: Charlson Comorbidity Index; CDDP: cisplatin; CIS: carcinoma in situ; CSS: cancer-specific survival; ECOG PS: Eastern Cooperative Oncology Group performance status; EPI: epirubicin; HCT: hematocrit; HGB: hemoglobin; IAC: intra-arterial chemotherapy; IQR: interquartile range; MMC: mitomycin C; NIAC: no-intraarterial chemotherapy; OS: overall survival; PLN: positive lymph nodes; PLND: pelvic lymph node dissection; RC: radical; WBC: white blood cell; GC: gemcitabine + cisplatin.; ST: survival time; CSR: cumulative survival rate

\section{Acknowledgements}

We wish to thank all our colleagues in the Department of Urology, Beijing Chao-Yang Hospital, without you, we could not have completed the work.

\section{Authors' contribution}

Author contributions: L.S., M.W. and F.Y. had full access to all of the data in the study and take responsibility for the integrity of the data and the 
accuracy of the data analysis. Acquisition of data: S.L., M.W. and Q.H. Study concept and design: Y.N. and N.X. Manuscript writing: W.W. and W.X. Statistical analysis: W.X. and L.H. Revision of the manuscript for intellectual content: Y.N. and N.X. Correspondence: Y.N. and N.X. All authors read and approved the final manuscript.

\section{Funding}

The study was funded by the Beijing Municipal Administration of Hospitals'Youth Programme (grant no. QML20160303) and the Beijing ChaoYang Hospital 1351 Talents Project Funding (grant no. CYXX-2017-11). The Foundation had no role in the design, implementation, data interpretation or manuscript preparation.

\section{Availability of data and materials}

The datasets used and/or analysed during the current study available from the corresponding author on request.

\section{Ethics approval and consent to participate}

This study was approved by the Institutional Review Board of Beijing ChaoYang Hospital (Project identification code: 2017-Science-71). Because more than half of our patients were from all over the country, follow-up and convalescence data were obtained by telephone contact. All participants were informed about the aims of this study and made the verbal consent.

\section{Consent for publication}

Not applicable.

\section{Competing interests}

The authors declare that they have no competing interests.

\section{Author details}

'Institute of Urology, Capital Medical University, Department of Urology, Capital Medical University Beijing Chao-Yang Hospital, Beijing 100020, China. ${ }^{2}$ Department of Urology, National Cancer Center/National Clinical Research Center for Cancer/Cancer Hospital, Chinese Academy of Medical Sciences and Peking Union Medical College, Beijing 100021, China. ${ }^{3}$ School of Biomedical Engineering, Capital Medical University, Beijing 100069, China.

Received: 6 July 2019 Accepted: 5 November 2019

\section{Published online: 16 November 2019}

\section{References}

1. Babjuk M, Bohle A, Burger M, Capoun O, Cohen D, Comperat EM, et al. EAU guidelines on non-muscle-invasive Urothelial carcinoma of the bladder: update 2016. Eur Urol. 2017;71(3):447-61.

2. Miller KD, Siegel RL, Lin CC, Mariotto AB, Kramer JL, Rowland JH, et al. Cancer treatment and survivorship statistics, 2016. CA Cancer J Clin. 2016; 66(4):271-89.

3. Stein JP, Lieskovsky G, Cote R, Groshen S, Feng AC, Boyd S, et al. Radical cystectomy in the treatment of invasive bladder cancer: long-term results in 1,054 patients. J Clin Oncol. 2001;19(3):666-75.

4. von der Maase H, Sengelov L, Roberts JT, Ricci S, Dogliotti L, Oliver T, et al Long-term survival results of a randomized trial comparing gemcitabine plus cisplatin, with methotrexate, vinblastine, doxorubicin, plus cisplatin in patients with bladder cancer. J Clin Oncol. 2005:23(21):4602-8.

5. Stein JP, Skinner DG. Radical cystectomy for invasive bladder cancer: longterm results of a standard procedure. World J Urol. 2006;24(3):296-304.

6. Dalbagni G, Genega E, Hashibe M, Zhang ZF, Russo P, Herr H, et al. Cystectomy for bladder cancer: a contemporary series. J Urol. 2001;165(4): 1111-6.

7. Bassi P, Ferrante GD, Piazza N, Spinadin R, Carando R, Pappagallo G, et al. Prognostic factors of outcome after radical cystectomy for bladder cancer: a retrospective study of a homogeneous patient cohort. J Urol. 1999;161(5): 1494-7.

8. Ghoneim MA. el-Mekresh MM, el-Baz MA, el-attar IA, Ashamallah a. radical cystectomy for carcinoma of the bladder: critical evaluation of the results in 1,026 cases. J Urol. 1997;158(2):393-9.

9. Donat SM, Shabsigh A, Savage C, Cronin AM, Bochner BH, Dalbagni G, et al. Potential impact of postoperative early complications on the timing of adjuvant chemotherapy in patients undergoing radical cystectomy: a highvolume tertiary cancer center experience. Eur Urol. 2009;55(1):177-85.
10. Thompson RH, Boorjian SA, Kim SP, Cheville JC, Thapa P, Tarrel R, et al. Eligibility for neoadjuvant/adjuvant cisplatin-based chemotherapy among radical cystectomy patients. BJU Int. 2014;113(5b):E17-21.

11. Galsky MD, Hahn NM, Rosenberg J, Sonpavde G, Hutson T, Oh WK, et al. Treatment of patients with metastatic urothelial cancer "unfit" for Cisplatinbased chemotherapy. J Clin Oncol. 2011;29(17):2432-8.

12. Reardon ZD, Patel SG, Zaid HB, Stimson CJ, Resnick MJ, Keegan KA, et al. Trends in the use of perioperative chemotherapy for localized and locally advanced muscle-invasive bladder cancer: a sign of changing tides. Eur Urol. 2015;67(1):165-70.

13. Kanoh S, Umeyama T, Nemoto S, Ishikawa S, Nemoto R, Rinsho K, et al. Long-term intra-arterial infusion chemotherapy with adriamycin for advanced bladder cancer. Cancer Chemother Pharmacol. 1983;11(Suppl): S51-8.

14. Kamidono S, Fujii A, Hamami G, Nakano Y, Umezu K, Oda Y, et al. New preoperative chemotherapy for bladder cancer using combination hemodialysis and direct hemoperfusion: preliminary report. J Urol. 1984; 131(1):36-40

15. Maatman TJ, Montie JE, Bukowski RM, Risius B, Geisinger M. Intra-arterial chemotherapy as an adjuvant to surgery in transitional cell carcinoma of the bladder. J Urol. 1986;135(2):256-60.

16. Kanoh S, Noguchi R, Ohtani M, Ishikawa S, Nemoto R, Koiso K, et al. Intraarterial chemotherapy for bladder cancer. Cancer Chemother Pharmacol. 1987;20(Suppl:S6-9).

17. Kakizaki H, Suzuki H, Kubota Y, Numasawa K, Suzuki K. Preoperative one-shot intra-arterial infusion chemotherapy for bladder cancer. Cancer Chemother Pharmacol. 1987;20(Suppl:S15-9).

18. Jacobs SC, Menashe DS, Mewissen MW, Lipchik EO. Intraarterial cisplatin infusion in the management of transitional cell carcinoma of the bladder. Cancer. 1989;64(2):388-91.

19. Galetti TP, Pontes JE, Montie J, Medendorp SV, Bukowski R. Neoadjuvant intra-arterial chemotherapy in the treatment of advanced transitional cell carcinoma of the bladder: results and followup. J Urol. 1989:142(5):1211-4 discussion 4-5.

20. Arima K, Tochigi H, Sugimura Y, Kawamura J. Balloon-occluded arterial infusion as a useful neoadjuvant chemotherapy for bladder cancer. $\mathrm{Br}$ J Urol. 1997:80(3):417-20.

21. Miyata Y, Nomata K, Ohba K, Matsuo T, Hayashi N, Sakamoto I, et al. Efficacy and safety of systemic chemotherapy and intra-arterial chemotherapy with/ without radiotherapy for bladder preservation or as neo-adjuvant therapy in patients with muscle-invasive bladder cancer: a single-Centre study of 163 patients. Eur J Surg Oncol. 2015;41(3):361-7.

22. Chism DD, Woods ME, Milowsky MI. Neoadjuvant paradigm for accelerated drug development: an ideal model in bladder cancer. Oncologist. 2013; 18(8):933-40.

23. Cancer Genome Atlas Research N. Comprehensive molecular characterization of urothelial bladder carcinoma. Nature. 2014;507(7492): 315-22.

24. Lawrence MS, Stojanov P, Polak P, Kryukov GV, Cibulskis K, Sivachenko A, et al. Mutational heterogeneity in cancer and the search for new cancerassociated genes. Nature. 2013;499(7457):214-8.

25. Siefker-Radtke AO, Apolo AB, Bivalacqua TJ, Spiess PE, Black PC. Immunotherapy with checkpoint blockade in the treatment of Urothelial carcinoma. J Urol. 2018;199(5):1129-42.

26. Krantz D, Hartana CA, Winerdal ME, Johansson M, Alamdari F, Jakubczyk T, et al. Neoadjuvant chemotherapy reinforces antitumour $T$ cell response in Urothelial urinary bladder Cancer. Eur Urol. 2018;74(6):688-92.

27. Necchi A, Anichini A, Raggi D, Briganti A, Massa S, Luciano R, et al. Pembrolizumab as Neoadjuvant Therapy Before Radical Cystectomy in Patients With Muscle-Invasive Urothelial Bladder Carcinoma (PURE-01): An Open-Label, Single-Arm, Phase II Study. J Clin Oncol. 2018:JCO1801148.

28. Tobert CM, Hamilton-Reeves JM, Norian LA, Hung C, Brooks NA, Holzbeierlein JM, et al. Emerging impact of malnutrition on surgical patients: literature review and potential implications for cystectomy in bladder Cancer. J Urol. 2017;198(3):511-9.

\section{Publisher's Note}

Springer Nature remains neutral with regard to jurisdictional claims in published maps and institutional affiliations. 